\title{
GAUSSIAN PROCESSES ASSOCIATED TO INFINITE BEAD-SPRING NETWORKS II: BEADS WITH MASS AND THE VANISHING MASS LIMIT*
}

\author{
MICHAEL TAYLOR ${ }^{\dagger}$
}

\begin{abstract}
We construct families of Gaussian processes $x_{\varepsilon}(t, n), t \in[0, \infty), n \in \mathbb{Z}$, modeling a class of infinite networks of stochastically fluctuating, interacting beads, of small mass, proportional to $\varepsilon$. We examine covariances $\mathbb{E}\left(x_{\varepsilon}\left(t_{1}, n_{1}\right) x_{\varepsilon}\left(t_{2}, n_{2}\right)\right)$ and draw conclusions about the subdiffusive nature of these processes, with particular attention to the behavior as $\varepsilon \rightarrow 0$. This complements previous work of the author, which in turn was influenced by work of McKinley, Yao, and Forest.
\end{abstract}

Key words. Gaussian processes, stochastic differential equations, singular perturbation.

AMS subject classifications. 60H30, 60H10, 60H05.

\section{Introduction}

In [7], following earlier work of [5] and [4], we studied the behavior of Gaussian processes that can be described as follows. Let $\ell^{2}(\mathbb{Z})$ denote the space of functions $a: \mathbb{Z} \rightarrow \mathbb{C}$ such that $\sum|a(n)|^{2}<\infty$ (here $\mathbb{Z}$ denotes the set of integers and $\mathbb{C}$ the set of complex numbers), and let $L$ be a negative semidefinite, self adjoint operator on $\ell^{2}(\mathbb{Z})$. We assume finitely supported elements of $\ell^{2}(\mathbb{Z})$ belong to the domain of $L$, so we can write

$$
L y(n)=\sum_{m \in \mathbb{Z}} \lambda(n, m) y(m) .
$$

Self adjointness implies $\lambda(n, m)=\overline{\lambda(m, n)}$. We assume

$$
\lambda(n, m) \in \mathbb{R}, \quad \text { hence } \lambda(m, n)=\lambda(n, m) .
$$

The process $x(t)=(x(t, n))$ studied in [7] solves the infinite system of stochastic differential equations

$$
d x(t, n)=L x(t, n) d t+\sigma d W_{n}(t), \quad x(0, n)=0
$$

for $n \in \mathbb{Z}, t \geq 0$. Here $W_{n}$ are independent, identically distributed Wiener processes. The system (1.3) provides a model for the motion of a polymer, pictured as a network of beads that interact and are also independently randomly jittered, as in Brownian motion. The particular case

$$
L y(n)=y(n-1)-2 y(n)+y(n+1)
$$

gives rise to what is called the Rouse chain model; see [5] and [4] for further details and references to the literature.

In [7], the solution to (1.3) was constructed in the form

$$
x(t, n)=\sigma \int_{0}^{t} \sum_{m \in \mathbb{Z}} h(t-s, n, m) d W_{m}(s),
$$

\footnotetext{
*Received: May 20, 2011; accepted (in revised version): August 26, 2011. Communicated by Shi Jin.

Work supported by NSF grant DMS-0758320.

${ }^{\dagger}$ Mathematics Deptartment, University of North Carolina, Chapel Hill NC 27599 USA (met@ math.unc.edu).
} 
where

$$
e^{t L} y(n)=\sum_{m} h(t, n, m) y(m) .
$$

It was shown that the series in (1.5) converges and defines a Gaussian process, with mean 0 . Formulas were derived for $\mathbb{E}\left(x\left(t_{1}, n_{1}\right) x\left(t_{2}, n_{2}\right)\right)$, with special consideration of

$$
\mathbb{E}\left(x(t, n)^{2}\right), \quad \text { and } \mathbb{E}\left(\left|x\left(t, n_{1}\right)-x\left(t, n_{2}\right)\right|^{2}\right) .
$$

The analysis of the first expectation in (1.7) recovered results of [5] and [4] on subdiffusivity of $x(t, n)$, and the analysis of the second expectation in (1.7), and also of $\mathbb{E}\left(x\left(t, n_{1}\right) x\left(t, n_{2}\right)\right)$, provided information on the joint distribution of $x\left(t, n_{1}\right)$ and $x\left(t, n_{2}\right)$.

As pointed out in [5] and [4], the system (1.3) is the $\varepsilon=0$ case of the second order system

$$
\varepsilon x_{\varepsilon}^{\prime \prime}(t, n)+x_{\varepsilon}^{\prime}(t, n)=L x_{\varepsilon}(t, n)+\sigma W_{n}^{\prime}(t),
$$

with prime denoting the $t$-derivative. Here $\varepsilon$ is proportional to the mass of each bead. It is reasonable to consider $\varepsilon$ to be positive but quite small. Thus it is of interest to study the solution $x_{\varepsilon}(t, n)$ to (1.8), with particular interest in the behavior as $\varepsilon \searrow 0$. This paper addresses that task. We take initial data

$$
x_{\varepsilon}(0, n)=0, \quad x_{\varepsilon}^{\prime}(0, n)=0, \quad \forall n \in \mathbb{Z} .
$$

Since (1.8) changes type when $\varepsilon$ reaches 0 , this is a singular perturbation problem. We first tackle it under an additional condition on $L$, namely that it be a bounded operator on $\ell^{2}(\mathbb{Z})$, with operator norm $\|L\|<\infty$. This condition holds for (1.4) and for many (arguably, for most) other examples arising in the bead-spring setting. Other examples include graph Laplacians, shown to be bounded in [7], in the case of infinite graphs, following results exposed for finite graphs in [1]. We produce a formula for the solution to (1.8)-(1.9) valid for

$$
0<\varepsilon<\frac{1}{4\|L\|},
$$

and study its behavior as $\varepsilon \searrow 0$. (In $\S 6$ we drop the hypothesis that $L$ be bounded and allow arbitrary $\varepsilon>0$.)

To see how such a formula arises, let us rewrite (1.5) as

$$
x(t)=\sigma \int_{0}^{t} e^{(t-s) L} d W(s),
$$

to celebrate how it comes from Duhamel's formula. To obtain an analogue for (1.8), we set $v_{\varepsilon}(t)=x_{\varepsilon}^{\prime}(t)$, i.e., $v_{\varepsilon}(t, n)=x_{\varepsilon}^{\prime}(t, n)$, and rewrite (1.8) as a first order system

$$
\frac{d}{d t}\left(\begin{array}{c}
x_{\varepsilon} \\
v_{\varepsilon}
\end{array}\right)=X_{\varepsilon}\left(\begin{array}{c}
x_{\varepsilon} \\
v_{\varepsilon}
\end{array}\right)+\left(\begin{array}{c}
0 \\
\beta \sigma W^{\prime}(t)
\end{array}\right)
$$

where

$$
X_{\varepsilon}=\left(\begin{array}{cc}
0 & I \\
\beta L & -\beta I
\end{array}\right)
$$


Here and below, we set

$$
\beta=\frac{1}{\varepsilon} .
$$

In (1.8) and (1.12), we use the "white noise" formalism $W^{\prime}(t)$. The system (1.12) is of course a Wiener-Itô stochastic differential equation, which can be written

$$
d\left(\begin{array}{c}
x_{\varepsilon} \\
v_{\varepsilon}
\end{array}\right)=X_{\varepsilon}\left(\begin{array}{c}
x_{\varepsilon} \\
v_{\varepsilon}
\end{array}\right) d t+\left(\begin{array}{c}
0 \\
\beta \sigma d W(t)
\end{array}\right)
$$

Taking into account the initial data (1.9), the Duhamel formula gives

$$
\begin{aligned}
\left(\begin{array}{l}
x_{\varepsilon}(t) \\
v_{\varepsilon}(t)
\end{array}\right) & =\sigma \int_{0}^{t} e^{(t-s) X_{\varepsilon}}\left(\begin{array}{c}
0 \\
\beta W^{\prime}(s)
\end{array}\right) d s \\
& =\sigma \int_{0}^{t} e^{(t-s) X_{\varepsilon}}\left(\begin{array}{c}
0 \\
\beta d W(s)
\end{array}\right) .
\end{aligned}
$$

To compute $e^{s X_{\varepsilon}}$, we note that by the spectral theorem (cf. [6], Chapter 7) we can treat $L$ as a real number and $X_{\varepsilon}$ as a real $2 \times 2$ matrix, with "eigenvalues"

$$
\lambda_{ \pm}(\beta, L)=-\frac{\beta}{2} I \pm \frac{\beta}{2}(I+4 \varepsilon L)^{1 / 2}
$$

and "eigenvectors"

$$
\left(\begin{array}{c}
1 \\
\lambda_{ \pm}(\beta, L)
\end{array}\right)
$$

One then calculates

$$
e^{t X_{\varepsilon}}\left(\begin{array}{l}
0 \\
1
\end{array}\right)=\left(\begin{array}{c}
\left(e^{t \lambda_{+}}-e^{t \lambda_{-}}\right) /\left(\lambda_{+}-\lambda_{-}\right) \\
\left(\lambda_{+} e^{t \lambda_{+}-} \lambda_{-} e^{t \lambda_{-}}\right) /\left(\lambda_{+}-\lambda_{-}\right)
\end{array}\right)
$$

Thus (1.15) yields

$$
x_{\varepsilon}(t)=\sigma \int_{0}^{t}\left[A_{\beta}^{+}(t-s)-A_{\beta}^{-}(t-s)\right] d W(s)
$$

where

$$
A_{\beta}^{ \pm}(s)=(I+4 \varepsilon L)^{-1 / 2} e^{s \lambda_{ \pm}(\beta, L)},
$$

and $\lambda_{ \pm}(\beta, L)$, given by (1.16), are bounded, negative semidefinite, self adjoint operators on $\ell^{2}(\mathbb{Z})$, as long as (1.10) holds. We have the task to show that the right side of (1.19) is a well defined Gaussian process and to investigate its properties, with particular attention to the behavior as $\varepsilon \searrow 0$, i.e., as $\beta \nearrow \infty$.

For use in subsequent sections, in $\S 2$ we collect some results on a class of vector stochastic integrals of the form

$$
x(t)=\int_{0}^{t} A(t-s) d W(s),
$$

where $\left\{A(s), A(s)^{*}: s \geq 0\right\}$ are strongly continuous families of bounded linear operators on $\ell^{2}(\mathbb{Z})$. Here, $x(t)=(x(t, n), n \in \mathbb{Z})$. We show that for each $n, x(t, n)$ is well defined 
and is a continuous function of $t \in[0, \infty)$ with values in $L^{2}(X, \nu)$, where $(X, \nu)$ is a naturally constructed probability space (see $\S 2$ for details). Also, for each $t \geq 0, n \in \mathbb{Z}$, $x(t, n)$ is a Gaussian random variable with mean zero. These results can be established via material in Chapter 4 of [2], but the setting here is more elementary. For the convenience of readers not familiar with infinite dimensional stochastic analysis, we give short, direct demonstrations of the needed formulas, as a consequence of classical work of Paley, Wiener, and Zygmund. Formulas established in $\S 2$ include

$$
\mathbb{E}\left(|x(t, n)|^{2}\right)=\int_{0}^{t}\left\|A(s)^{*} \delta_{n}\right\|_{\ell^{2}}^{2} d s
$$

and more generally

$$
\mathbb{E}\left(x\left(t, n_{1}\right) \overline{x\left(t, n_{2}\right)}\right)=\int_{0}^{t}\left(A(s)^{*} \delta_{n_{2}}, A(s)^{*} \delta_{n_{1}}\right) d s,
$$

where $\left\{\delta_{n}: n \in \mathbb{Z}\right\}$ is the orthonormal basis of $\ell^{2}(\mathbb{Z})$ given by $\delta_{n}(m)=1$ if $m=n, 0$ otherwise. If $A(s)$ and $A(s)^{*}$ commute for all $s$, one can erase the asterisks in (1.22) and (1.23).

In $\S 3$ we apply the results of $\S 2$ to $A(s)=A_{\beta}^{ \pm}(s)$, given by (1.20), and construct

$$
x_{\varepsilon}(t, n)=x_{\varepsilon}^{+}(t, n)-x_{\varepsilon}^{-}(t, n),
$$

when (1.10) holds. Here $x_{\varepsilon}^{ \pm}(t, n)$ is the $n$th component of

$$
x_{\varepsilon}^{ \pm}(t)=\sigma \int_{0}^{t} A_{\beta}^{ \pm}(t-s) d W(s)
$$

with $A_{\beta}^{ \pm}$as in (1.20). We compare $x_{\varepsilon}(t, n)$ to the solution to (1.3), given by (1.5), which we now denote $x_{0}(t, n)$. We show that

$$
\mathbb{E}\left(\left|x_{\varepsilon}^{+}(t, n)-x_{0}(t, n)\right|^{2}\right) \leq C \varepsilon \mathbb{E}\left(x_{0}(t, n)^{2}\right),
$$

and

$$
\mathbb{E}\left(x_{\varepsilon}^{-}(t, n)^{2}\right) \leq C \sigma^{2}\left(1-e^{-t / \varepsilon}\right) \varepsilon,
$$

provided $0<\varepsilon \leq a /\|L\|$, with $a<1 / 4$; see Theorem 3.1. These estimates imply that whenever $x_{0}(t, n)$ is subdiffusive, i.e.,

$$
\frac{1}{t} \mathbb{E}\left(x_{0}(t, n)^{2}\right) \longrightarrow 0 \text { as } t \nearrow \infty,
$$

the processes $x_{\varepsilon}(t, n)$ are uniformly subdiffusive, for $\varepsilon$ in such an interval.

In $\S 4$ we note that the processes $x_{\varepsilon}^{ \pm}(t, n)$ are differentiable (as functions of $t$ with values in $\left.L^{2}(X, \nu)\right)$, for $\varepsilon$ satisfying (1.10), and study

$$
v_{\varepsilon}^{ \pm}(t, n)=\frac{d}{d t} x_{\varepsilon}^{ \pm}(t, n) .
$$

At least one of these must blow up as $\varepsilon \searrow 0$, since $x_{0}(t, n)$ is not differentiable; as it turns out, $v_{\varepsilon}^{-}(t, n)$ blows up. We show that

$$
\mathbb{E}\left(v_{\varepsilon}^{+}(t, n)^{2}\right) \leq C \mathbb{E}\left(x_{0}(t, n)^{2}\right),
$$


but

$$
\mathbb{E}\left(v_{\varepsilon}^{-}(t, n)^{2}\right) \geq \frac{\sigma^{2}}{4 \varepsilon}\left(1-e^{-t / \varepsilon}\right) .
$$

In $\S 5$ we convert formulas for $\mathbb{E}\left(x_{\varepsilon}(t, n)^{2}\right)$ into integral formulas, arising from a spectral representation of $L$, and examine the asymptotic behavior as $t \nearrow \varnothing$, including more precise versions of the subdiffusivity result (1.27) and their counterparts for $\mathbb{E}\left(x_{\varepsilon}(t, n)^{2}\right)$; see Theorems 5.1-5.2.

Results of $\S \S 3-5$ use the hypothesis (1.10). We obtain estimates valid uniformly for $0<\varepsilon \leq a /\|L\|$, given $a<1 / 4$. In $\S 6$ we extend the scope of our investigation, in two ways. First, we replace (1.10) by

$$
0<\varepsilon<\infty
$$

Second, we remove the hypothesis that $L$ be bounded. In this more general setting, frequently $-1 / 4 \varepsilon$ belongs to the spectrum of $L$ and represents a transition from overdamping to underdamping in the system (1.8). The operators $A_{\beta}^{ \pm}(s)$ in (1.20) are then not bounded, and the processes $x_{\varepsilon}^{ \pm}(t, n)$ do not exist. However,

$$
A_{\beta}(s)=A_{\beta}^{+}(s)-A_{\beta}^{-}(s)
$$

is bounded. In fact, from (1.20) we obtain

$$
A_{\beta}(s)=s \beta e^{-s \beta / 2} H\left(\frac{s \beta}{2}(I+4 \varepsilon L)^{1 / 2}\right),
$$

where $H$ is the entire holomorphic, even function on $\mathbb{C}$ given by

$$
H(z)=\frac{\sinh z}{z}, \quad H(0)=1 .
$$

Using this, we show that the processes $x_{\varepsilon}(t, n)$ exist. We obtain formulas for $\mathbb{E}\left(x_{\varepsilon}(t, n)^{2}\right)$, etc., extending those obtained earlier for $\varepsilon$ satisfying (1.10). Making use of these results, we extend the scope of results of $\S 5$. Our main results in this section are given in Theorems 6.1-6.2.

\section{A class of vector stochastic integrals}

In this section we provide some useful formulas for vector stochastic integrals of the form

$$
x(t)=\int_{0}^{t} A(t-s) d W(s)
$$

where, for each $s \geq 0$,

$$
A(s): \ell^{2}(\mathbb{Z}) \longrightarrow \ell^{2}(\mathbb{Z})
$$

is a bounded linear operator. For simplicity we assume

$$
A(s) \text { and } A(s)^{*} \text { are strongly continuous in } s \in[0, \infty),
$$

though the calculations below will make it clear that we can relax this hypothesis. Written out more fully, (2.1) takes the form

$$
x(t, n)=\int_{0}^{t} \sum_{m \in \mathbb{Z}} a(t-s, n, m) d W_{m}(s),
$$


where, for $y \in \ell^{2}(\mathbb{Z})$,

$$
A(s) y(n)=\sum_{m} a(s, n, m) y(m)
$$

The operators arising in (1.19) are self adjoint (for $0<\varepsilon<1 / 4\|L\|$ ) and reality preserving, but we do not need these properties for the development here. Consequently, the processes (2.4) might be complex valued. Note the adjoint $A(s)^{*}$ of $A(s)$ satisfies

$$
A(s)^{*} y(m)=\sum_{m} a^{*}(s, n, m) y(m), \quad a^{*}(s, n, m)=\overline{a(s, m, n)},
$$

and that

$$
a(s, n, m)=A(s) \delta_{m}(n), \quad a^{*}(s, n, m)=A(s)^{*} \delta_{m}(n),
$$

where $\delta_{n} \in \ell^{2}(\mathbb{Z})$ is given by

$$
\delta_{n}(m)=\delta_{n, m}= \begin{cases}1 & \text { if } m=n \\ 0 & \text { otherwise }\end{cases}
$$

As stated in the introduction, $\left\{W_{n}: n \in \mathbb{Z}\right\}$ is a collection of independent, identically distributed Wiener processes. In more detail, let $B(t)$ be the Wiener process (Brownian motion), which is a continuous family $B(t) \in L^{2}(\Omega, \mu)$, where $\Omega$ is path space and $\mu$ is Wiener measure. Then set $\Omega_{n}=\Omega, \mu_{n}=\mu$, for $n \in \mathbb{Z}$, and take the product space (with product measure)

$$
(X, \nu)=\prod_{n \in \mathbb{Z}}\left(\Omega_{n}, \mu_{n}\right) .
$$

We obtain (2.4) as

$$
x(t, n)=\lim _{K \rightarrow \infty} \sum_{m=-K}^{K} \xi_{m}(t, n),
$$

where

$$
\xi_{m}(t, n)=\int_{0}^{t} a(t-s, n, m) d W_{m}(s) .
$$

Our first task is to establish convergence in $L^{2}(X, \nu)$ of the right side of $(2.10)$. Note that

$$
m \neq m^{\prime} \Longrightarrow \xi_{m}(t, n) \perp \xi_{m^{\prime}}(t, n) \text { in } L^{2}(X, \nu),
$$

so it suffices to bound $\sum_{m} \mathbb{E}\left(\left|\xi_{m}(t, n)\right|^{2}\right)$. To get this, note that

$$
\mathbb{E}\left(\left|\xi_{m}(t, n)\right|^{2}\right)=\int_{0}^{t} a(s, n, m) a^{*}(s, m, n) d s,
$$

which is the classical Paley-Wiener-Zygmund identity (cf. [3], §2.1). Hence

$$
\sum_{m} \mathbb{E}\left(\left|\xi_{m}(t, n)\right|^{2}\right)=\sum_{m} \int_{0}^{t} a(s, n, m) a^{*}(s, m, n) d s
$$




$$
\begin{aligned}
& =\sum_{m} \int_{0}^{t} a(s, n, m) A(s)^{*} \delta_{n}(m) d s \\
& =\int_{0}^{t} A(s) A(s)^{*} \delta_{n}(n) d s \\
& =\int_{0}^{t}\left(A(s) A(s)^{*} \delta_{n}, \delta_{n}\right) d s \\
& =\int_{0}^{t}\left\|A(s)^{*} \delta_{n}\right\|_{\ell^{2}}^{2} d s .
\end{aligned}
$$

Here $\delta_{n} \in \ell^{2}(\mathbb{Z})$ is given by (2.8). Thus we have convergence in (2.10), and

$$
\mathbb{E}\left(|x(t, n)|^{2}\right)=\int_{0}^{t}\left\|A(s)^{*} \delta_{n}\right\|_{\ell^{2}}^{2} d s
$$

The nature of the convergence implies that for each $n \in \mathbb{Z}, t \geq 0, x(t, n)$ is a Gaussian random variable on $(X, \nu)$ with mean 0 .

We next aim to show that, under the hypotheses in $(2.3), x(t, n)$ is a continuous function of $t \in[0, \infty)$ with values in $L^{2}(X, \nu)$, for each $n$. In preparation for this, we note that

$$
\mathbb{E}\left(x\left(t_{1}, n\right) \overline{x\left(t_{2}, n\right)}\right)=\sum_{k} \int_{0}^{t_{1} \wedge t_{2}} a\left(t_{1}-s, n, k\right) a^{*}\left(t_{2}-s, k, n\right) d s .
$$

Here $t_{1} \wedge t_{2}=\min \left(t_{1}, t_{2}\right)$. We have

$$
\begin{aligned}
\sum_{k} a\left(t_{1}-s, n, k\right) a^{*}\left(t_{2}-s, k, n\right) & =\sum_{k} a\left(t_{1}-s, n, k\right) A\left(t_{2}-s\right)^{*} \delta_{n}(k) \\
& =A\left(t_{1}-a\right) A\left(t_{2}-s\right)^{*} \delta_{n}(n) \\
& =\left(A\left(t_{2}-s\right)^{*} \delta_{n}, A\left(t_{1}-s\right)^{*} \delta_{n}\right),
\end{aligned}
$$

so

$$
\mathbb{E}\left(x\left(t_{1}, n\right), \overline{x\left(t_{2}, n\right)}\right)=\int_{0}^{t_{1} \wedge t_{2}}\left(A\left(t_{2}-s\right)^{*} \delta_{n}, A\left(t_{1}-s\right)^{*} \delta_{n}\right) d s .
$$

Now

$$
\begin{aligned}
& \mathbb{E}\left(\left|x\left(t_{1}, n\right)-x\left(t_{2}, n\right)\right|^{2}\right) \\
= & \mathbb{E}\left(x\left(t_{1}, n\right)^{2}\right)+\mathbb{E}\left(x\left(t_{2}, n\right)^{2}\right)-2 \operatorname{Re} \mathbb{E}\left(x\left(t_{1}, n\right) \overline{x\left(t_{2}, n\right)}\right),
\end{aligned}
$$

so (2.18) gives (say if $\left.0 \leq t_{1} \leq t_{2}\right)$

$$
\begin{aligned}
& \mathbb{E}\left(\left|x\left(t_{1}, n\right)-x\left(t_{2}, n\right)\right|^{2}\right) \\
= & \int_{t_{1}}^{t_{2}}\left\|A\left(t_{2}-s\right)^{*} \delta_{n}\right\|_{\ell^{2}}^{2} d s+\int_{0}^{t_{1} \wedge t_{2}}\left\{\left(A\left(t_{1}-s\right)^{*} \delta_{n}, A\left(t_{1}-s\right)^{*} \delta_{n}\right)\right. \\
& \left.+\left(A\left(t_{2}-s\right)^{*} \delta_{n}, A\left(t_{2}-s\right)^{*} \delta_{n}\right)-2 \operatorname{Re}\left(A\left(t_{2}-s\right)^{*} \delta_{n}, A\left(t_{1}-s\right)^{*} \delta_{n}\right)\right\} d s .
\end{aligned}
$$

The first integral on the right side of $(2.20)$ is $\leq C\left|t_{1}-t_{2}\right|$. We can write the second integral as

$$
\begin{aligned}
& \operatorname{Re} \int_{0}^{t_{1} \wedge t_{2}}\left\{\left(\left[A\left(t_{1}-s\right)^{*}-A\left(t_{2}-s\right)^{*}\right] \delta_{n}, A\left(t_{1}-s\right)^{*} \delta_{n}\right)\right. \\
& \left.+\left(A\left(t_{2}-s\right)^{*} \delta_{n},\left[A\left(t_{2}-s\right)^{*}-A\left(t_{1}-s\right)^{*}\right] \delta_{n}\right)\right\} d s .
\end{aligned}
$$


Given (2.3), the fact that (2.21) tends to 0 as $t_{1} \rightarrow t_{2}$ follows readily from the Lebesgue dominated convergence theorem. Let us summarize what we have established.

Proposition 2.1. Under the hypotheses (2.2)-(2.3), the formula (2.4) gives for each $n \in \mathbb{Z}$ a well defined $x(t, n)$, a continuous function of $t \in[0, \infty)$ with values in $L^{2}(X, \nu)$, for each $t, n$ a Gaussian randon variable with mean 0, satisfying the identities (2.15) and (2.18).

We complement (2.15) with the following computation, derived similarly.

$$
\begin{aligned}
\mathbb{E}\left(x\left(t, n_{1}\right) \overline{x\left(t, n_{2}\right)}\right) & =\sum_{k} \int_{0}^{t} a\left(t-s, n_{1}, k\right) \overline{a\left(t-s, n_{2}, k\right)} d s \\
& =\sum_{k} \int_{0}^{t} a\left(t-s, n_{1}, k\right) a^{*}\left(t-s, k, n_{2}\right) d s .
\end{aligned}
$$

Parallel to (2.17), we then get

$$
\begin{aligned}
\mathbb{E}\left(x\left(t, n_{1}\right) \overline{x\left(t, n_{2}\right)}\right) & =\int_{0}^{t}\left(A(t-s)^{*} \delta_{n_{2}}, A(t-s)^{*} \delta_{n_{1}}\right) d s \\
& =\int_{0}^{t}\left(A(s)^{*} \delta_{n_{2}}, A(s)^{*} \delta_{n_{1}}\right) d s .
\end{aligned}
$$

Combining (2.15) and (2.23), we have

$$
\begin{aligned}
& \mathbb{E}\left(\left|x\left(t, n_{1}\right)-x\left(t, n_{2}\right)\right|^{2}\right) \\
= & \mathbb{E}\left(\left|x\left(t, n_{1}\right)\right|^{2}\right)+\mathbb{E}\left(\left|x\left(t, n_{2}\right)\right|^{2}\right)-2 \operatorname{Re} \mathbb{E}\left(x\left(t, n_{1}\right) \overline{x\left(t, n_{2}\right)}\right) \\
= & \int_{0}^{t}\left\{\left\|A(s)^{*} \delta_{n_{1}}\right\|_{\ell^{2}}^{2}+\left\|A(s)^{*} \delta_{n_{2}}\right\|_{\ell^{2}}^{2}-2 \operatorname{Re}\left(A(s)^{*} \delta_{n_{2}}, A(s)^{*} \delta_{n_{1}}\right)\right\} d s \\
= & \int_{0}^{t}\left\|A(s)^{*}\left(\delta_{n_{1}}-\delta_{n_{2}}\right)\right\|_{\ell^{2}}^{2} d s .
\end{aligned}
$$

We now give a condition under which the components $x(t, n)$ of the process $(2.1)$ are differentiable, as functions of $t$ with values in $L^{2}(X, \nu)$. Let us add to $(2.3)$ the hypothesis

$$
A^{\prime}(s) \text { and } A^{\prime}(s)^{*} \text { are strongly continuous in } s \in[0, \infty) .
$$

Then, as in the scalar case, Wiener's integration by parts formula holds for (2.1):

$$
x(t)=\int_{0}^{t} A^{\prime}(t-s) W(s) d s+A(0) W(t) .
$$

We have the following.

Proposition 2.2. In the setting of Proposition 2.1, if also (2.25) holds and $A(0)=0$, then $x(t, n)$ is differentiable for each $n \in \mathbb{Z}$, and $x^{\prime}(t, n)$ is a continuous function of $t \in[0, \infty)$ with values in $L^{2}(X, \nu)$.

Proof. Let us temporarily assume that (2.25) also holds for $A^{\prime \prime}(s)$. Then we differentiate $(2.26)$ and get (provided $A(0)=0)$

$$
x^{\prime}(t)=\int_{0}^{t} A^{\prime \prime}(t-s) W(s) d s+A^{\prime}(0) W(t) .
$$


Applying (2.26) with $A$ replaced by $A^{\prime}$ then gives

$$
x^{\prime}(t)=\int_{0}^{t} A^{\prime}(t-s) d W(s) .
$$

A mollification and approximation argument gives (2.28) without the additional assumption on $A^{\prime \prime}$.

Returning to the computations (2.13)-(2.23), note that if $A(s)$ is self adjoint for all $s$, all the asterisks can be removed, and if these operators are reality preserving, all the overlines can be removed. Furthermore,

$$
\begin{aligned}
& A(s)^{*} A(s)=A(s) A(s)^{*} \\
& \Longrightarrow\left\|A(s)^{*} \delta_{n}\right\|_{\ell^{2}}=\left\|A(s) \delta_{n}\right\|_{\ell^{2}} \text {, and } \\
& \left(A(s)^{*} \delta_{n_{2}}, A(s)^{*} \delta_{n_{1}}\right)=\left(A(s) \delta_{n_{2}}, A(s) \delta_{n_{1}}\right),
\end{aligned}
$$

so we have the following.

Proposition 2.3. In the setting of Proposition 2.1, if also $A(s)$ is normal for all $s \geq 0$, then

$$
\mathbb{E}\left(|x(t, n)|^{2}\right)=\int_{0}^{t}\left\|A(s) \delta_{n}\right\|_{\ell^{2}}^{2} d s
$$

and more generally

$$
\mathbb{E}\left(x\left(t, n_{1}\right) \overline{x\left(t, n_{2}\right)}\right)=\int_{0}^{t}\left(A(s) \delta_{n_{2}}, A(s) \delta_{n_{1}}\right) d s .
$$

\section{The processes $x_{\varepsilon}(t, n)$}

Results of $\S 2$ on the vector stochastic integral $\int_{0}^{t} A(t-s) d W(s)$ apply to (1.19) with

$$
\begin{aligned}
A(s) & =\sigma\left[A_{\beta}^{+}(s)-A_{\beta}^{-}(s)\right], \\
A_{\beta}^{ \pm}(s) & =(I+4 \varepsilon L)^{-1 / 2} e^{s \lambda_{ \pm}(\beta, L),} \\
\lambda_{ \pm}(\beta, L) & =-\frac{\beta}{2} I \pm \frac{\beta}{2}(I+4 \varepsilon L)^{1 / 2} .
\end{aligned}
$$

In the current setting, $L$ is a bounded, reality preserving, negative semidefinite, self adjoint operator on $\ell^{2}(\mathbb{Z}), 0<\varepsilon<1 / 4\|L\|$, and $\beta=1 / \varepsilon$. Hence $\lambda_{ \pm}(\beta, L)$ are negative semidefinite, self adjoint operators on $\ell^{2}(\mathbb{Z})$. Thus, for each such $\varepsilon, x_{\varepsilon}(t)=$ $\left(x_{\varepsilon}(t, n), n \in \mathbb{Z}\right)$ has the property that, for each $n \in \mathbb{Z}, x_{\varepsilon}(t, n)$ is a continuous function of $t \in[0, \infty)$ with values in $L^{2}(X, \nu)$, and for each $t \geq 0$ is a real valued Gaussian random variable with mean 0. For further analysis, it is convenient (using (1.19)) to write

$$
x_{\varepsilon}(t, n)=x_{\varepsilon}^{+}(t, n)-x_{\varepsilon}^{-}(t, n),
$$

where

$$
x_{\varepsilon}^{ \pm}(t)=\sigma \int_{0}^{t} A_{\beta}^{ \pm}(t-s) d W(s) .
$$


The formula (2.15) gives

$$
\mathbb{E}\left(x_{\varepsilon}^{ \pm}(t, n)^{2}\right)=\sigma^{2} \int_{0}^{t}\left\|A_{\beta}^{ \pm}(s) \delta_{n}\right\|_{\ell^{2}}^{2} d s .
$$

Note that Spec $\lambda_{-}(\beta, L) \subset(-\infty,-\beta / 2]$, so we have the operator norm estimate

$$
\left\|A_{\beta}^{-}(s)\right\| \leq\left\|(I+4 \varepsilon L)^{-1 / 2}\right\| e^{-s \beta / 2},
$$

and we get

$$
\mathbb{E}\left(x_{\varepsilon}^{-}(t, n)^{2}\right) \leq C \sigma^{2} \varepsilon\left(1-e^{-\beta t}\right), \quad 0<\varepsilon \leq \frac{a}{\|L\|}, \quad a<\frac{1}{4},
$$

with $C$ independent of $t \in[0, \infty)$.

In order to analyze $x_{\varepsilon}^{+}(t, n)$, note that, as long as (1.10) holds,

$$
(I+4 \varepsilon L)^{1 / 2}=I+2 \varepsilon L \Phi(4 \varepsilon L)
$$

with $\Phi(\lambda)$ given by

$$
\begin{aligned}
(1+\lambda)^{1 / 2} & =1+\frac{1}{2} \lambda-\frac{1}{8} \lambda^{2}+\cdots \\
& =1+\frac{1}{2} \lambda\left(1-\frac{1}{4} \lambda+\cdots\right) \\
& =1+\frac{1}{2} \lambda \Phi(\lambda) .
\end{aligned}
$$

Note that $\Phi(\lambda)$ is holomorphic on $\{\lambda \in \mathbb{C}:|\lambda|<1\}$ and

$$
\Phi(0)=1, \quad \Phi(\lambda)>0 \text { for } \lambda \in(-1,1) .
$$

Hence

$$
\lambda_{+}(\beta, L)=L \Phi(4 \varepsilon L)
$$

so

$$
A_{\beta}^{+}(s)=(I+4 \varepsilon L)^{-1 / 2} e^{s L \Phi(4 \varepsilon L)},
$$

and

$$
x_{\varepsilon}^{+}(t)=\sigma \int_{0}^{t}(I+4 \varepsilon L)^{-1 / 2} e^{(t-s) L \Phi(4 \varepsilon L)} d W(s) .
$$

Hence

$$
\begin{aligned}
\mathbb{E}\left(x_{\varepsilon}^{+}(t, n)^{2}\right) & =\sigma^{2} \int_{0}^{t}\left\|(I+4 \varepsilon L)^{-1 / 2} e^{s L \Phi(4 \varepsilon L)} \delta_{n}\right\|_{\ell^{2}}^{2} d s \\
& =\sigma^{2} \int_{0}^{t}\left(e^{2 s L \Phi(4 \varepsilon L)}(I+4 \varepsilon L)^{-1} \delta_{n}, \delta_{n}\right) d s .
\end{aligned}
$$

If we set

$$
G(\lambda)=\int_{0}^{1} e^{-s \lambda} d s= \begin{cases}\frac{1-e^{-\lambda}}{\lambda} & , \lambda>0 \\ 1 \quad & , \lambda=0\end{cases}
$$


we can write (3.13) as

$$
\mathbb{E}\left(x_{\varepsilon}^{+}(t, n)^{2}\right)=\sigma^{2} t\left(G(-2 t L \Phi(4 \varepsilon L))(I+4 \varepsilon L)^{-1} \delta_{n}, \delta_{n}\right) .
$$

In $\S 5$ we will investigate large $t$ behavior of this.

At this point, it is natural to compare $x_{\varepsilon}^{+}(t)$ with the solution $x_{0}(t)$ to (1.3), given by (1.11), i.e.,

$$
x_{0}(t)=\sigma \int_{0}^{t} e^{(t-s) L} d W(s) .
$$

Note that, parallel to (3.13)-(3.15),

$$
\begin{aligned}
\mathbb{E}\left(x_{0}(t, n)^{2}\right) & =\sigma^{2} \int_{0}^{t}\left\|e^{s L} \delta_{n}\right\|_{\ell^{2}}^{2} d s \\
& =\sigma^{2} t\left(G(-2 t L) \delta_{n}, \delta_{n}\right) .
\end{aligned}
$$

Applying (2.15) to the difference of (3.12) and (3.16) gives

$$
\begin{aligned}
& \mathbb{E}\left(\left|x_{\varepsilon}^{+}(t, n)-x_{0}(t, n)\right|^{2}\right) \\
= & \sigma^{2} \int_{0}^{t}\left\|\left[A_{\beta}^{+}(t-s)-e^{(t-s) L}\right] \delta_{n}\right\|_{\ell^{2}}^{2} d s \\
= & \sigma^{2} \int_{0}^{t}\left\|\left[(I+4 \varepsilon L)^{-1 / 2} e^{s L \Phi(4 \varepsilon L)}-e^{s L}\right] \delta_{n}\right\|_{\ell^{2}}^{2} d s .
\end{aligned}
$$

Using $(a+b)^{2} \leq 2 a^{2}+2 b^{2}$, we can write

$$
\mathbb{E}\left(\left|x_{\varepsilon}^{+}(t, n)-x_{0}(t, n)\right|^{2}\right) \leq 2 \sigma^{2}\left(A_{\varepsilon}(t, n)+B_{\varepsilon}(t, n)\right),
$$

where

$$
\begin{aligned}
& A_{\varepsilon}(t, n)=\int_{0}^{t}\left\|(I+4 \varepsilon L)^{-1 / 2}\left[e^{s L \Phi(4 \varepsilon L)}-e^{s L}\right] \delta_{n}\right\|_{\ell^{2}}^{2} d s, \\
& B_{\varepsilon}(t, n)=\int_{0}^{t}\left\|\left[(I+4 \varepsilon L)^{-1 / 2}-I\right] e^{s L} \delta_{n}\right\|_{\ell^{2}}^{2} d s .
\end{aligned}
$$

Noting that

$$
\left\|(I+4 \varepsilon L)^{-1 / 2}-I\right\| \leq C \varepsilon, \quad \text { for } \quad 0<\varepsilon \leq \frac{a}{\|L\|}, \quad a<\frac{1}{4},
$$

and comparing (3.17), we have

$$
\sigma^{2} B_{\varepsilon}(t, n) \leq C \varepsilon \mathbb{E}\left(x_{0}(t, n)^{2}\right) .
$$

We also have

$$
A_{\varepsilon}(t, n) \leq C \widetilde{A}_{\varepsilon}(t, n),
$$

where

$$
\widetilde{A}_{\varepsilon}(t, n)=\int_{0}^{t}\left\|\left[e^{s L \Phi(4 \varepsilon L)}-e^{s L}\right] \delta_{n}\right\|_{\ell^{2}}^{2} d s
$$


To proceed, recall from (3.8) that

$$
(1+\lambda)^{1 / 2}=1+\frac{1}{2} \lambda \Phi(\lambda)
$$

where $\Phi(\lambda)$ is given by

$$
\Phi(\lambda)=1-\frac{1}{4} \lambda+\cdots=1-\frac{\lambda}{4} \psi(\lambda), \quad \psi(0)=1,
$$

with $\psi(\lambda)$ holomorphic in $\{\lambda \in \mathbb{C}:|\lambda|<1\}$, real and positive for $\lambda \in(-1,1)$. The positivity can be seen from the concavity of $(1+\lambda)^{1 / 2}$, which implies $(1+\lambda)^{1 / 2} \leq 1+\lambda / 2$ on $(-1,1)$, hence $\Phi(\lambda) \leq 1$ on $[0,1)$ and $\geq 1$ on $(-1,0]$. Hence

$$
e^{s L \Phi(4 \varepsilon L)}-e^{s L}=\left(e^{-s \varepsilon L^{2} \psi(4 \varepsilon L)}-I\right) e^{s L},
$$

and we have

$$
\widetilde{A}_{\varepsilon}(t, n)=\int_{0}^{t}\left\|\left(I-e^{-s \varepsilon L^{2} \psi(4 \varepsilon L)}\right) e^{s L} \delta_{n}\right\|_{\ell^{2}}^{2} d s
$$

which gives

$$
\sigma^{2} \widetilde{A}_{\varepsilon}(t, n) \leq \sup _{0<s<t}\left\|I-e^{-s \varepsilon L^{2} \psi(4 \varepsilon L)}\right\|^{2} \mathbb{E}\left(x_{0}(t, n)^{2}\right) .
$$

If we take $a \in(0,1 / 4)$ and set

$$
\alpha=\sup _{0<\varepsilon \leq a /\|L\|}\left\|L^{2} \psi(4 \varepsilon L)\right\|
$$

then, since $L^{2} \psi(4 \varepsilon L)$ is positive semidefinite, we have

$$
\sigma^{2} \widetilde{A}_{\varepsilon}(t, n) \leq\left(1-e^{-\alpha \varepsilon t}\right)^{2} \mathbb{E}\left(x_{0}(t, n)^{2}\right),
$$

provided

$$
0<\varepsilon \leq \frac{a}{\|L\|}, \quad a<\frac{1}{4} .
$$

The factor in front of $\mathbb{E}\left(x_{0}(t, n)^{2}\right)$ in $(3.31)$ is $O(\varepsilon)$ for $t$ in each bounded interval in $[0, \infty)$, but one loses uniformity as $t \nearrow \infty$. In fact, $(3.31)$ is not optimal. We proceed to derive a stronger estimate. Writing

$$
\left(I-e^{-s \varepsilon L^{2} \psi(4 \varepsilon L)}\right) e^{s L}=\left(e^{s L / 2}-e^{s L / 2-s \varepsilon L^{2} \psi(4 \varepsilon L)}\right) e^{s L / 2}
$$

we have

$$
\widetilde{A}_{\varepsilon}(t, n) \leq A_{\varepsilon}^{\#}(t)^{2} \int_{0}^{t}\left\|e^{s L / 2} \delta_{n}\right\|_{\ell^{2}}^{2} d s
$$

with

$$
\begin{aligned}
A_{\varepsilon}^{\#}(t) & =\sup _{0<s<t}\left\|e^{s L / 2}-e^{s L / 2-s \varepsilon L^{2} \psi(4 \varepsilon L)}\right\| \\
& \leq \sup _{0<s<t, 0 \leq \Lambda \leq\|L\|}\left|e^{-s \Lambda / 2}-e^{-s \Lambda / 2-s \varepsilon \Lambda^{2} \psi(-4 \varepsilon \Lambda)}\right|
\end{aligned}
$$


the latter inequality by the spectral theorem. Now, over the range $0 \leq \Lambda \leq\|L\|$,

$$
\varphi=\Lambda \psi(-4 \varepsilon \Lambda) \Longrightarrow 0 \leq \varphi \leq \Lambda_{0},
$$

as long as (3.32) holds, where $\Lambda_{0}=\|L\| \sup _{(-1,1)} \psi(\lambda)$, and we obtain

$$
A_{\varepsilon}^{\#}(t) \leq \sup _{0<s<t, \Lambda \geq 0,0 \leq \varphi \leq \Lambda_{0}}\left|e^{-s \Lambda / 2}-e^{-s \Lambda / 2-s \varepsilon \Lambda \varphi}\right| .
$$

Taking $s \Lambda \mapsto \Lambda$, we get

$$
\begin{aligned}
A_{\varepsilon}^{\#}(t) & \leq \sup _{\Lambda \geq 0,0 \leq \varphi \leq \Lambda_{0}}\left|e^{-\Lambda / 2}-e^{-\Lambda / 2-\varepsilon \varphi \Lambda}\right| \\
& \leq \sup _{\Lambda \geq 0,0 \leq \varphi \leq \Lambda_{0}} \varepsilon \varphi \Lambda e^{-\Lambda / 2} \\
& \leq \varepsilon \Lambda_{0},
\end{aligned}
$$

since $\sup \Lambda e^{-\Lambda / 2}=2 / e<1$. Note that this estimate is independent of $t$. Meanwhile,

$$
\begin{aligned}
\sigma^{2} \int_{0}^{t}\left\|e^{s L / 2} \delta_{n}\right\|_{\ell^{2}}^{2} d s & =2 \sigma^{2} \int_{0}^{t / 2}\left\|e^{s L} \delta_{n}\right\|_{\ell^{2}}^{2} d s \\
& =2 \mathbb{E}\left(x_{0}\left(\frac{t}{2}, n\right)^{2}\right) \\
& \leq 2 \mathbb{E}\left(x_{0}(t, n)^{2}\right),
\end{aligned}
$$

so (3.34) and (3.38) yield

$$
\sigma^{2} \widetilde{A}_{\varepsilon}(t, n) \leq 2 \Lambda_{0} \varepsilon \mathbb{E}\left(x_{0}(t, n)^{2}\right) .
$$

Let us collect the main results established above.

Theorem 3.1. As long as (3.32) holds, the formulas (3.2)-(3.3) give, for each $n \in \mathbb{Z}$, a mean zero Gaussian process $t \mapsto x_{\varepsilon}(t, n)=x_{\varepsilon}^{+}(t, n)-x_{\varepsilon}^{-}(t, n)$, a continuous function of $t \in[0, \infty)$ with values in $L^{2}(X, \nu)$. Furthermore, there exist $C, \alpha \in(0, \infty)$ such that when (3.32) holds and $x_{0}(t, n)$ is given by (3.16), then for all $n \in \mathbb{Z}, t \geq 0$,

$$
\mathbb{E}\left(\left|x_{\varepsilon}^{+}(t, n)-x_{0}(t, n)\right|^{2}\right) \leq C \varepsilon \mathbb{E}\left(x_{0}(t, n)^{2}\right),
$$

and

$$
\mathbb{E}\left(x_{\varepsilon}^{-}(t, n)^{2}\right) \leq C \sigma^{2}\left(1-e^{-t / \varepsilon}\right) \varepsilon .
$$

We record formulas for the covariance of $x_{\varepsilon}^{ \pm}\left(t, n_{1}\right)$ and $x_{\varepsilon}^{ \pm}\left(t, n_{2}\right)$. By $(2.23)$, we have (with coherent choice of signs)

$$
\begin{aligned}
& \mathbb{E}\left(x_{\varepsilon}^{ \pm}\left(t, n_{1}\right) x_{\varepsilon}^{ \pm}\left(t, n_{2}\right)\right) \\
= & \sigma^{2} \int_{0}^{t}\left(A_{\beta}^{ \pm}(s) \delta_{n_{1}}, A_{\beta}^{ \pm}(s) \delta_{n_{2}}\right) \\
= & \sigma^{2} \int_{0}^{t}\left((I+4 \varepsilon L)^{-1} e^{2 s \lambda_{ \pm}(\beta, L)} \delta_{n_{1}}, \delta_{n_{2}}\right) d s
\end{aligned}
$$

In particular, using (3.10),

$$
\begin{aligned}
& \mathbb{E}\left(x_{\varepsilon}^{+}\left(t, n_{1}\right) x_{\varepsilon}^{+}\left(t, n_{2}\right)\right) \\
= & \sigma^{2} \int_{0}^{t}\left((I+4 \varepsilon L)^{-1} e^{2 s L \Phi(4 \varepsilon L)} \delta_{n_{1}}, \delta_{n_{2}}\right) d s \\
= & \sigma^{2} t\left((I+4 \varepsilon L)^{-1} G(-2 t L \Phi(4 \varepsilon L)) \delta_{n_{1}}, \delta_{n_{2}}\right) .
\end{aligned}
$$


4. The processes $v_{\varepsilon}(t, n)$

From $(3.1)$ we see that $A_{\beta}^{ \pm}(0)=(I+4 \varepsilon L)^{-1 / 2}$ for $0<\varepsilon<1 / 4\|L\|$, so $A(0)=0$ and, by Proposition $2.2, x_{\varepsilon}(t, n)$ is differentiable, as a function of $t$, with values in $L^{2}(X, \nu)$, for each $n \in \mathbb{Z}$. By (2.28),

$$
x_{\varepsilon}^{\prime}(t, n)=v_{\varepsilon}(t, n)=v_{\varepsilon}^{+}(t, n)-v_{\varepsilon}^{-}(t, n),
$$

with

$$
v_{\varepsilon}^{ \pm}(t)=\sigma \int_{0}^{t} V_{\beta}^{ \pm}(t-s) d W(s)
$$

where

$$
\begin{aligned}
V_{\beta}^{ \pm}(s) & =\frac{d}{d s} A_{\beta}^{ \pm}(s) \\
& =(I+4 \varepsilon L)^{-1 / 2} \lambda_{ \pm}(\beta, L) e^{s \lambda_{ \pm}(\beta, L)} .
\end{aligned}
$$

As before, $\beta=1 / \varepsilon$. We will compute square expectations and verify, as one should expect, that $\mathbb{E}\left(v_{\varepsilon}(t, n)^{2}\right) \rightarrow \infty$ as $\varepsilon \searrow 0$. In fact, we separately examine $\mathbb{E}\left(v_{\varepsilon}^{+}(t, n)^{2}\right)$ and $\mathbb{E}\left(v_{\varepsilon}^{-}(t, n)^{2}\right)$, and see that only the latter blows up as $\varepsilon \searrow 0$.

To begin, we have

$$
\begin{aligned}
& \mathbb{E}\left(v_{\varepsilon}^{ \pm}(t, n)^{2}\right) \\
= & \sigma^{2} \int_{0}^{t}\left\|V_{\beta}^{ \pm}(s) \delta_{n}\right\|_{\ell^{2}}^{2} d s \\
= & \sigma^{2} \int_{0}^{t}\left\|(I+4 \varepsilon L)^{-1 / 2} \lambda_{ \pm}(\beta, L) e^{s \lambda_{ \pm}(\beta, L)} \delta_{n}\right\|_{\ell^{2}}^{2} d s .
\end{aligned}
$$

Recalling from (3.10) that $\lambda_{+}(\beta, L)=L \Phi(4 \varepsilon L)$, we have, for

$$
0<\varepsilon \leq \frac{a}{\|L\|}, \quad a<\frac{1}{4},
$$

that

$$
\begin{aligned}
& \mathbb{E}\left(v_{\varepsilon}^{+}(t, n)^{2}\right) \\
= & \sigma^{2} \int_{0}^{t}\left\|L \Phi(4 \varepsilon L)(I+4 \varepsilon L)^{-1 / 2} e^{s L \Phi(4 \varepsilon L)} \delta_{n}\right\|_{\ell^{2}}^{2} d s \\
\leq & C \mathbb{E}\left(x_{\varepsilon}^{+}(t, n)^{2}\right) \\
\leq & C^{\prime} \mathbb{E}\left(x_{0}(t, n)^{2}\right),
\end{aligned}
$$

the first inequality by (3.13), given the operator norm bound $\|L \Phi(4 \varepsilon L)\| \leq C$, and the second by (3.41).

To proceed, we have

$$
\begin{aligned}
& \mathbb{E}\left(v_{\varepsilon}^{-}(t, n)^{2}\right) \\
= & \sigma^{2} \int_{0}^{t}\left((I+4 \varepsilon L)^{-1} \lambda_{-}(\beta, L)^{2} e^{2 s \lambda_{-}(\beta, L)} \delta_{n}, \delta_{n}\right) d s \\
= & -\frac{\sigma^{2}}{2}\left((I+4 \varepsilon L)^{-1} \lambda_{-}(\beta, L)\left(I-e^{2 t \lambda_{-}(\beta, L)}\right) \delta_{n}, \delta_{n}\right) .
\end{aligned}
$$


Now, as long as (4.5) holds, we have, via the spectral theorem,

$$
\begin{aligned}
\operatorname{Spec}(I+4 \varepsilon L)^{-1} & \subset[1, \infty), \\
\operatorname{Spec} \lambda_{-}(\beta, L) & \subset[-\beta,-\beta / 2], \\
\operatorname{Spec}\left(I-e^{2 t \lambda_{-}(\beta, L)}\right) & \subset\left[1-e^{-\beta t}, 1\right],
\end{aligned}
$$

and hence

$$
\operatorname{Spec}-(I+4 \varepsilon L)^{-1} \lambda_{-}(\beta, L)\left(I-e^{2 t \lambda_{-}(\beta, L)}\right) \subset\left[\frac{\beta}{2}\left(1-e^{-t \beta}\right), \infty\right) .
$$

The variational characterization of the bottom of the spectrum for a positive definite, self adjoint operator, applied to the last inner product in (4.7), then gives

$$
\mathbb{E}\left(v_{\varepsilon}^{-}(t, n)^{2}\right) \geq \frac{\sigma^{2}}{4 \varepsilon}\left(1-e^{-t / \varepsilon}\right),
$$

as long as (4.5) holds. The right side of (4.10) clearly blows up as $\varepsilon \searrow 0$, for each $t>0$.

\section{Spectral representation, asymptotics, and subdiffusivity}

Let $L: \ell^{2}(\mathbb{Z}) \rightarrow \ell^{2}(\mathbb{Z})$ be a bounded, negative, self adjoint operator, as described in $\S 1$. The spectral theorem (cf. [6], Theorem VII.3) implies there is a measure space $(S, \gamma)$, a unitary map

$$
\mathcal{F}: \ell^{2}(\mathbb{Z}) \longrightarrow L^{2}(S, \gamma)
$$

and a function

$$
\Lambda \in L^{\infty}(S, \gamma), \quad \Lambda \geq 0, \quad\|\Lambda\|_{L^{\infty}}=\|L\|,
$$

such that for each $y \in \ell^{2}(\mathbb{Z}), t \geq 0$,

$$
\mathcal{F} L y(\theta)=-\Lambda(\theta) \mathcal{F} y(\theta), \quad \theta \in S .
$$

Consequently,

$$
\begin{aligned}
\mathcal{F} e^{t L} y(\theta) & =e^{-t \Lambda(\theta)} \mathcal{F} y(\theta), \\
\mathcal{F} \Phi(4 \varepsilon L) y(\theta) & =\Phi(-4 \varepsilon \Lambda(\theta)) \mathcal{F} y(\theta),
\end{aligned}
$$

etc. The orthonormal basis $\left\{\delta_{n}\right\}$ of $\ell^{2}(\mathbb{Z})$ gives rise to the orthonormal basis $\left\{e_{n}\right\}$ of $L^{2}(S, \gamma)$,

$$
e_{n}=\mathcal{F} \delta_{n}
$$

Using these ingredients, we can rewrite the formula (3.17) for the square expectation of $x_{0}(t, n)$ as

$$
\begin{aligned}
\mathbb{E}\left(x_{0}(t, n)^{2}\right) & =\sigma^{2} \int_{S} \int_{0}^{t} e^{-2 s \Lambda(\theta)}\left|e_{n}(\theta)\right|^{2} d s d \gamma(\theta) \\
& =\sigma^{2} t \int_{S} G(2 t \Lambda(\theta))\left|e_{n}(\theta)\right|^{2} d \gamma(\theta) .
\end{aligned}
$$


Similarly, (3.13)-(3.15) yield

$$
\begin{aligned}
& \mathbb{E}\left(x_{\varepsilon}^{+}(t, n)^{2}\right) \\
= & \sigma^{2} \int_{S} \int_{0}^{t}(1-4 \varepsilon \Lambda(\theta))^{-1} e^{-2 s \Lambda_{\varepsilon}(\theta)}\left|e_{n}(\theta)\right|^{2} d s d \gamma(\theta) \\
= & \sigma^{2} t \int_{S}(1-4 \varepsilon \Lambda(\theta))^{-1} G\left(2 t \Lambda_{\varepsilon}(\theta)\right)\left|e_{n}(\theta)\right|^{2} d \gamma(\theta),
\end{aligned}
$$

for

$$
0<\varepsilon<\frac{1}{4\|L\|}
$$

Here,

$$
\begin{aligned}
\Lambda_{\varepsilon}(\theta) & =\Lambda(\theta) \Phi(-4 \varepsilon \Lambda(\theta)) \\
& =\Lambda(\theta)(1+\varepsilon \Lambda(\theta) \psi(-4 \varepsilon \Lambda(\theta))),
\end{aligned}
$$

with $\Phi$ as in (3.7)-(3.10) and $\psi$ as in (3.26). Note that, as long as (5.8) holds, $\psi(-4 \varepsilon \Lambda(\theta)) \geq 0$. More generally, by (3.44),

$$
\begin{aligned}
& \mathbb{E}\left(x_{\varepsilon}^{+}\left(t, n_{1}\right) x_{\varepsilon}^{+}\left(t, n_{2}\right)\right) \\
= & \sigma^{2} \int_{S} \int_{0}^{t}(1-4 \varepsilon \Lambda(\theta))^{-1} e^{-2 s \Lambda_{\varepsilon}(\theta)} e_{n_{1}}(\theta) \overline{e_{n_{2}}(\theta)} d s d \gamma(\theta) \\
= & \sigma^{2} t \int_{S}(1-4 \varepsilon \Lambda(\theta))^{-1} G\left(2 t \Lambda_{\varepsilon}(\theta)\right) e_{n_{1}}(\theta) \overline{e_{n_{2}}(\theta)} d \gamma(\theta) .
\end{aligned}
$$

Let us specialize to the case that $L$ is of convolution type:

$$
L y(n)=\sum_{m} \lambda(n-m) y(m) .
$$

A special case is given in (1.4), for the Rouse chain model. The convolution case was also emphasized in [5] and [4]. In this case, we can take

$$
\begin{gathered}
S=S^{1}=\mathbb{R} /(2 \pi \mathbb{Z}), \quad d \gamma(\theta)=d \theta / 2 \pi, \\
\mathcal{F} y(\theta)=\hat{y}(\theta)=\sum_{n} y(n) e^{i n \theta}, \quad e_{n}(\theta)=e^{i n \theta}, \quad \Lambda(\theta)=-\hat{\lambda}(\theta) .
\end{gathered}
$$

In such a case, (5.6)-(5.10) become

$$
\begin{gathered}
\mathbb{E}\left(x_{0}(t, n)^{2}\right)=\frac{\sigma^{2} t}{2 \pi} \int_{S^{1}} G(2 t \Lambda(\theta)) d \theta, \\
\mathbb{E}\left(x_{\varepsilon}^{+}(t, n)^{2}\right)=\frac{\sigma^{2} t}{2 \pi} \int_{S^{1}}(1-4 \varepsilon \Lambda(\theta))^{-1} G\left(2 t \Lambda_{\varepsilon}(\theta)\right) d \theta,
\end{gathered}
$$


and

$$
\begin{aligned}
& \mathbb{E}\left(x_{\varepsilon}^{+}\left(t, n_{1}\right) x_{\varepsilon}^{+}\left(t, n_{2}\right)\right) \\
= & \frac{\sigma^{2} t}{2 \pi} \int_{S^{1}}(1-4 \varepsilon \Lambda(\theta))^{-1} G\left(2 t \Lambda_{\varepsilon}(\theta)\right) e^{i\left(n_{1}-n_{2}\right) \theta} d \theta .
\end{aligned}
$$

Note that the reality condition (1.2) implies

$$
\lambda(-n)=\lambda(n), \text { hence } \Lambda(-\theta)=\Lambda(\theta) .
$$

Taking this into account, a short computation yields

$$
\begin{aligned}
& \mathbb{E}\left(\left|x_{\varepsilon}^{+}\left(t, n_{1}\right)-x_{\varepsilon}^{+}\left(t, n_{2}\right)\right|^{2}\right) \\
= & \mathbb{E}\left(x_{\varepsilon}^{+}\left(t, n_{1}\right)^{2}\right)+\mathbb{E}\left(x_{\varepsilon}^{+}\left(t, n_{2}\right)^{2}\right)-2 \mathbb{E}\left(x_{\varepsilon}^{+}\left(t, n_{1}\right) x_{\varepsilon}^{+}\left(t, n_{2}\right)\right) \\
= & \frac{4 \sigma^{2} t}{2 \pi} \int_{S^{1}}(1-4 \varepsilon \Lambda(\theta))^{-1} G\left(2 t \Lambda_{\varepsilon}(\theta)\right) \sin ^{2} \frac{\left(n_{1}-n_{2}\right) \theta}{2} d \theta .
\end{aligned}
$$

Similarly (as seen in [7]), we have

$$
\begin{aligned}
& \mathbb{E}\left(\left|x_{0}\left(t, n_{1}\right)-x_{0}\left(t, n_{2}\right)\right|^{2}\right) \\
= & \frac{4 \sigma^{2} t}{2 \pi} \int_{S^{1}} G(2 t \Lambda(\theta)) \sin ^{2} \frac{\left(n_{1}-n_{2}\right) \theta}{2} d \theta .
\end{aligned}
$$

Note that for the Rouse chain model, where $L$ is given by (1.4), we have (5.11) with

$$
\lambda(n)= \begin{cases}-2, & n=0 \\ 1, & n= \pm 1 \\ 0, & \text { otherwise }\end{cases}
$$

and hence

$$
\Lambda(\theta)=2-e^{i \theta}-e^{-i \theta}=4 \sin ^{2} \frac{\theta}{2} .
$$

In (2.16) of [4] it was shown that if $\Lambda(\theta)$ is smooth and $>0$ on $S^{1} \backslash\{0\}$ and

$$
\Lambda(\theta) \sim|\theta|^{\rho} \sum_{k \geq 0} a_{k}|\theta|^{k}, \quad \theta \rightarrow 0,
$$

with $a_{0} \neq 0$, then

$$
\frac{t}{2 \pi} \int_{S^{1}} G(2 t \Lambda(\theta)) d \theta \sim \begin{cases}C t^{1-1 / \rho}, & \rho>1, \\ C \log t, & \rho=1, \\ C, & \rho \in(0,1)\end{cases}
$$

as $t \rightarrow \infty$, and consequently, by (5.13),

$$
\mathbb{E}\left(x_{0}(t, n)^{2}\right) \sim \sigma^{2} \times \text { right side of (5.22), as } t \rightarrow \infty .
$$


This applies to (5.20) with $\rho=2$. This large $t$ behavior is to be contrasted with that of the Wiener process:

$$
\mathbb{E}\left(W_{n}(t)^{2}\right)=t
$$

Because (5.23) is significantly smaller than (5.24) for large $t$, one says the process $x_{0}(t, n)$ is subdiffusive. This subdiffusivity result was supplemented in [7] by the following (Propositions 4.1 and 6.1 of [7]), whose proof follows readily from (5.6) and the Lebesgue dominated convergence theorem.

Proposition 5.1. In the general setting of (5.1)-(5.6), if

$$
\Lambda(\theta)>0 \text { for } \gamma \text {-a.e. } \theta \in S
$$

then, for each $n \in \mathbb{Z}$,

$$
\mathbb{E}\left(x_{0}(t, n)^{2}\right)=o(t) \text { as } t \rightarrow \infty
$$

Applying Theorem 3.1 immediately leads to the following extension of this result.

THEOREM 5.1. In the general setting of (5.1)-(5.5), if (5.25) holds, then, for each $n \in \mathbb{Z}$,

$$
\mathbb{E}\left(x_{\varepsilon}(t, n)^{2}\right)=o(t) \quad \text { as } t \rightarrow \infty,
$$

uniformly in $\varepsilon \in(0, a /\|L\|]$, for each $a<1 / 4$.

Similarly, Theorem 3.1 yields the following extension of the subdiffusivity results for $x_{0}(t, n)$ discussed above.

THEOREM 5.2. In the setting of (5.11)-(5.12), if $\Lambda(\theta)$ is smooth and $>0$ on $S^{1} \backslash\{0\}$, and satisfies (5.21), then

$$
\mathbb{E}\left(x_{\varepsilon}(t, n)^{2}\right) \leq \begin{cases}C t^{1-1 / \rho}, & \rho>1, \\ C \log t, & \rho=1, \\ C, & \rho \in(0,1),\end{cases}
$$

uniformly for

$$
0<\varepsilon \leq \frac{a}{\|L\|}, \quad a<\frac{1}{4}
$$

The condition (5.29) will be relaxed in $\S 6$.

REMARK 5.3. In (5.28) we have estimates, as opposed to the asymptotic result in (5.23). To obtain a uniform asymptotic analysis of $\mathbb{E}\left(x_{\varepsilon}(t, n)^{2}\right)$ is an intriguing problem, which we hope to take up in future work. 


\section{Extension of the scope}

In this section, we discard the restriction (1.10) on $\varepsilon$ and allow arbitrary $\varepsilon>0$. As always, $L$ is a negative semidefinite, self adjoint operator on $\ell^{2}(\mathbb{Z})$, but here we do not require $L$ to be bounded. We will assume that finitely supported elements of $\ell^{2}(\mathbb{Z})$ belong to the domain of $L$. As mentioned in the introduction, the operators $A_{\beta}^{ \pm}(s)$, given by (1.20), need not be bounded. On the other hand, we have

$$
\begin{aligned}
A_{\beta}(s) & =A_{\beta}^{+}(s)-A_{\beta}^{-}(s) \\
& =(I+4 \varepsilon L)^{-1 / 2}\left[e^{s \lambda_{+}(\beta, L)}-e^{s \lambda_{-}(\beta, L)}\right] \\
& =(I+4 \varepsilon L)^{-1 / 2}\left[e^{(s \beta / 2)(I+4 \varepsilon L)^{1 / 2}}-e^{-(s \beta / 2)(I+4 \varepsilon L)^{1 / 2}}\right] e^{-s \beta / 2} \\
& =s \beta e^{-s \beta / 2} H\left(\frac{s \beta}{2}(I+4 \varepsilon L)^{1 / 2}\right),
\end{aligned}
$$

where

$$
H(z)=\frac{\sinh z}{z}, \quad H(0)=1 .
$$

Note that $H(z)$ is an entire function, even in $z$. There can be some ambiguity in specifying $(I+4 \varepsilon L)^{1 / 2}$, but the fact that $H(z)$ is even in $z$ makes such ambiguity harmless. We have $\operatorname{Spec}(I+4 \varepsilon L)^{1 / 2} \subset(0,1]$ if $(1.10)$ holds, while if we merely have $\varepsilon>0$, we can say

$$
\operatorname{Spec}(I+4 \varepsilon L)^{1 / 2} \subset[0,1] \cup i \mathbb{R}
$$

Note that for $x, y \in \mathbb{R}$,

$$
H(x)=\frac{\sinh x}{x} \text { and } H(i y)=\frac{\sin y}{y}
$$

are real. Hence, for $A_{\beta}(s)$ as in (6.1), we have

$$
A_{\beta}(s)^{*}=A_{\beta}(s) .
$$

To estimate the operator norm of $A_{\beta}(s)$, note that $|\sin y| \leq|y|$ for $y \in \mathbb{R}$, and a calculation gives $H^{\prime}(x)>0$ for $x \in[0, \infty)$, so

$$
\sup \left\{|H(z)|: z \in\left[0, \frac{s \beta}{2}\right] \cup i \mathbb{R}\right\}=H\left(\frac{s \beta}{2}\right) .
$$

Consequently,

$$
\begin{aligned}
\left\|A_{\beta}(s)\right\| & \leq s \beta e^{-s \beta / 2} \frac{\sinh (s \beta / 2)}{s \beta / 2} \\
& =1-e^{-s \beta},
\end{aligned}
$$

with equality if (as happens in the interesting cases) $0 \in \operatorname{Spec} L$.

Results of $\S 2$ imply the processes $x_{\varepsilon}(t)=\left(x_{\varepsilon}(t, n): n \in \mathbb{Z}\right)$ given by (1.19) are well defined for all $\varepsilon>0$. If we allow $L$ to be unbounded, we need to note that (6.1) gives a strongly continuous family of operators on $\ell^{2}(\mathbb{Z})$.

Note that

$$
A_{\beta}^{\prime}(s)=-\frac{\beta}{2} A_{\beta}(s)+\beta e^{-s \beta / 2} \cosh \left(\frac{s \beta}{2}(I+4 \varepsilon L)^{1 / 2}\right),
$$


so, extending results of $\S 4$, we have $x_{\varepsilon}(t, n)$ differentiable for all $\varepsilon>0$, as a function of $t$ with values in $L^{2}(X, \nu)$.

As in $\S 5$, the spectral theorem produces a unitary map

$$
\mathcal{F}: \ell^{2}(\mathbb{Z}) \longrightarrow L^{2}(S, \gamma)
$$

and a measurable function

$$
\Lambda: S \longrightarrow[0, \infty)
$$

(not bounded if $L$ is not bounded), such that

$$
\mathcal{F} e^{t L} y(\theta)=e^{-t \Lambda(\theta)} \mathcal{F} y(\theta) .
$$

In place of (5.7), we have

$$
\begin{aligned}
& \mathbb{E}\left(x_{\varepsilon}(t, n)^{2}\right) \\
= & \sigma^{2} \int_{0}^{t}\left\|A_{\beta}(s) \delta_{n}\right\|_{\ell^{2}}^{2} d s \\
= & \sigma^{2} \int_{0}^{t} \int_{S}(s \beta)^{2} e^{-s \beta} H\left(\frac{s \beta}{2}(1-4 \varepsilon \Lambda(\theta))^{1 / 2}\right)^{2}\left|e_{n}(\theta)\right|^{2} d \gamma(\theta) d s,
\end{aligned}
$$

where $e_{n}=\mathcal{F} \delta_{n}$. Similarly,

$$
\begin{aligned}
& \mathbb{E}\left(\left|x_{\varepsilon}(t, n)-x_{0}(t, n)\right|^{2}\right) \\
= & \sigma^{2} \int_{0}^{t} \int_{S}\left[e^{-s \Lambda(\theta)}-s \beta e^{-s \beta / 2} H\left(\frac{s \beta}{2}(1-4 \varepsilon \Lambda(\theta))^{1 / 2}\right)\right]^{2}\left|e_{n}(\theta)\right|^{2} d \gamma(\theta) d s .
\end{aligned}
$$

Calculations parallel to those done in $\S 3$ establish that

$$
\begin{aligned}
& \theta \in S, \Lambda(\theta)<\infty, s \in[0, \infty), \beta=\varepsilon^{-1} \\
& \Longrightarrow \lim _{\varepsilon \searrow 0} s \beta e^{-s \beta / 2} H\left(\frac{s \beta}{2}(1-4 \varepsilon \Lambda(\theta))^{1 / 2}\right)=e^{-s \Lambda(\theta)} .
\end{aligned}
$$

Also, by (6.6)-(6.7), the integrand in (6.13) is dominated in absolute value by $4\left|e_{n}(\theta)\right|^{2}$, so the Lebesgue dominated convergence theorem establishes the following.

Proposition 6.1. In the current setting, for each $t \in[0, \infty), n \in \mathbb{Z}$,

$$
\lim _{\varepsilon \searrow 0} \mathbb{E}\left(\left|x_{\varepsilon}(t, n)-x_{0}(t, n)\right|^{2}\right)=0 .
$$

This is a partial extension of Theorem 3.1, though it lacks the punch of the estimates (3.41)-(3.42). We aim to sharpen this up.

To proceed let us fix $M \in[1, \infty)$, take

$$
\varepsilon \in\left(0, \frac{M}{4}\right]
$$

and set

$$
S_{a}=\left\{\theta \in S: \Lambda(\theta) \leq \frac{1}{2 M}\right\}, \quad S_{b}=S \backslash S_{a} .
$$


Thus $\varepsilon \Lambda(\theta) \geq \varepsilon / 2 M$ on $S_{b}$, so

$$
\theta \in S_{b} \Rightarrow(1-4 \varepsilon \Lambda(\theta))^{1 / 2} \begin{cases}\leq 1-\frac{\varepsilon}{M} & \text { if } 4 \varepsilon \Lambda(\theta) \leq 1, \\ \text { is purely imaginary } & \text { if } 4 \varepsilon \Lambda(\theta) \geq 1 .\end{cases}
$$

Making use of (6.6), with $s \beta / 2$ replaced by $(s \beta / 2)(1-\varepsilon / M)$, we have

$$
\begin{aligned}
\theta \in S_{b} \Rightarrow & s \beta e^{-s \beta / 2}\left|H\left(\frac{s \beta}{2}(1-4 \varepsilon \Lambda(\theta))^{1 / 2}\right)\right| \\
\leq & s \beta e^{-s \beta / 2} H\left(\frac{s \beta}{2}\left(1-\frac{\varepsilon}{M}\right)\right) \\
& =\frac{2 e^{-s \beta / 2}}{1-\varepsilon / M}\left(e^{(s \beta / 2)(1-\varepsilon / M)}-e^{-(s \beta / 2)(1-\varepsilon / M)}\right) \\
& =\frac{2}{1-\varepsilon / M}\left(e^{-s / 2 M}-e^{-s \beta(1-\varepsilon / 2 M)}\right) \\
\leq & 4 e^{-s / 2 M},
\end{aligned}
$$

the second identity via $\varepsilon \beta=1$. In addition,

$$
\theta \in S_{b} \Longrightarrow e^{-s \Lambda(\theta)} \leq e^{-s / 2 M}
$$

so, if $I(s, \varepsilon, \theta)$ denotes the integrand in (6.13), we have

$$
I(s, \varepsilon, \theta) \leq 25 e^{-s / M}\left|e_{n}(\theta)\right|^{2}, \quad \forall \theta \in S_{b},
$$

and hence

$$
\begin{aligned}
\sigma^{2} \int_{0}^{t} \int_{S_{b}} I(s, \varepsilon, \theta) d \gamma(\theta) d s & \leq 25 M \sigma^{2} \int_{S_{b}}\left|e_{n}(\theta)\right|^{2} d \gamma(\theta) \\
& \leq 25 M \sigma^{2}
\end{aligned}
$$

so as $\varepsilon \rightarrow 0$ this contribution to (6.13) converges to 0 with uniform bounds, independent of $t$.

Next, for $\theta \in S_{a}$, write

$$
\begin{aligned}
& s \beta e^{-s \beta / 2} H\left(\frac{s \beta}{2}(1-4 \varepsilon \Lambda(\theta))^{1 / 2}\right) \\
= & \frac{e^{-(s \beta / 2)+(s \beta / 2)(1-4 \varepsilon \Lambda(\theta))^{1 / 2}}}{(1-4 \varepsilon \Lambda(\theta))^{1 / 2}}-\frac{e^{-(s \beta / 2)-(s \beta / 2)(1-4 \varepsilon \Lambda(\theta))^{1 / 2}}}{(1-4 \varepsilon \Lambda(\theta))^{1 / 2}} .
\end{aligned}
$$

We have $4 \varepsilon \Lambda(\theta) \leq 2 \varepsilon / M \leq 1 / 2$ on $S_{a}$, given that $\varepsilon$ satisfies (6.16), so the last term in $(6.23)$ is

$$
\leq \sqrt{2} e^{-s \beta / 2} \text { on } S_{a} .
$$

Thus, with $I(s, \varepsilon, \theta)$ as in (6.21)-(6.22), we have

$$
\sigma^{2} \int_{0}^{t} \int_{S_{a}} I(s, \varepsilon, \theta) d \gamma(\theta) d s
$$




$$
\begin{aligned}
& =\sigma^{2} \int_{0}^{t} \int_{S_{a}}\left[e^{-s \Lambda(\theta)}-\frac{e^{-(s \beta / 2)+(s \beta / 2)(1-4 \varepsilon \Lambda(\theta))^{1 / 2}}}{(1-4 \varepsilon \Lambda(\theta))^{1 / 2}}\right]^{2}\left|e_{n}(\theta)\right|^{2} d \gamma(\theta) d s \\
& \quad+R(t, \varepsilon),
\end{aligned}
$$

where

$$
|R(t, \varepsilon)| \leq C \int_{0}^{t} e^{-s \beta} d s \leq C \varepsilon,
$$

with $C$ independent of $t$. Next, estimates parallel to (3.18)-(3.40) apply to the main term on the right side of (6.25), given that $\varepsilon \leq M / 4$ and $\Lambda(\theta) \leq 1 / 2 M$. We have the main term

$$
\leq C \sigma^{2} \mathbb{E}\left(x_{0}(t, n)^{2}\right) \varepsilon .
$$

Putting together these estimates, we have the following.

Theorem 6.2. For each $M \in[1, \infty)$, we have $C<\infty$ such that, as long as $0<\varepsilon \leq M / 4$,

$$
\mathbb{E}\left(\left|x_{\varepsilon}(t, n)-x_{0}(t, n)\right|^{2}\right) \leq C \sigma^{2} \mathbb{E}\left(x_{0}(t, n)^{2}\right) \varepsilon+C \varepsilon+R_{b}(\varepsilon, t),
$$

with

$$
R_{b}(\varepsilon, t) \leq 25 M \sigma^{2}, \quad \forall t \geq 0,
$$

and

$$
\lim _{\varepsilon \rightarrow 0} R_{b}(\varepsilon, t)=0
$$

Using Theorem 6.2 in place of Theorem 3.1, we have the following extension of Theorem 5.2.

THEOREM 6.3. In the setting of (5.11)-(5.12), if $\Lambda(\theta)$ is smooth and $>0$ on $S^{1} \backslash\{0\}$, and satisfies (5.21), then (5.28) holds, uniformly for $\varepsilon \in(0, K]$, for each $K<\infty$.

\section{REFERENCES}

[1] F. Chung, Spectral Graph Theory, CBMS Reg. Conf. Ser. Math. 92, American Mathematical Society, Providence, RI, 1997.

[2] G. DaPrato and J. Zabczyk, Stochastic Equations in Infinite Dimensions, Encl. Math. Appl., Cambridge Univ. Press, Cambridge, 45, 1992.

[3] H. McKean, Stochastic Integrals, Academic Press, New York, 1969.

[4] S. McKinley, Anomalous diffusion of distinguished particles in bead-spring networks, preprint, 2009, arXiv:0911.4293.

[5] S. McKinley, L. Yao, and M. G. Forest, Transient anomalous diffusion of tracer particles in soft matter, J. Rheology, 53, 2009.

[6] M. Reed and B. Simon, Methods of Mathematical Physics, Academic Press, New York, 1, 1980.

[7] M. Taylor, Gaussian processes associated to infinite bead-spring networks, Commun. Math. Sci., 9, 517-534, 2011. 